\title{
Edge localized mode control with an edge resonant magnetic perturbation ${ }^{\text {a) }}$
}

\author{
R. A. Moyer, ${ }^{2, b)}$ T. E. Evans ${ }^{1}$ T. H. Osborne, ${ }^{1}$ P. R. Thomas,${ }^{3}$ M. Becoulet, ${ }^{3}$ J. Harris, ${ }^{4}$ \\ K.-H. Finken, ${ }^{5}$ J. A. Boedo, ${ }^{2}$ E. J. Doyle, ${ }^{6}$ M. E. Fenstermacher, ${ }^{7}$ P. Gohil, ${ }^{1}$ \\ R. J. Groebner, ${ }^{1}$ M. Groth, ${ }^{7}$ G. L. Jackson, ${ }^{1}$ R. J. La Haye, ${ }^{1}$ C. J. Lasnier, ${ }^{7}$ A. W. Leonard, ${ }^{1}$ \\ G. R. McKee, ${ }^{8}$ H. Reimerdes, ${ }^{9}$ T. L. Rhodes, ${ }^{6}$ D. L. Rudakov, ${ }^{2}$ M. J. Schaffer, ${ }^{1}$ \\ P. B. Snyder, ${ }^{1}$ M. R. Wade, ${ }^{10}$ G. Wang, ${ }^{6}$ J. G. Watkins, ${ }^{11}$ W. P. West, ${ }^{1}$ and L. Zeng ${ }^{6}$ \\ ${ }_{1}^{1}$ General Atomics, P.O. Box 85608, San Diego, California 92186-5688 \\ ${ }^{2}$ University of California, San Diego, La Jolla, California 92093-0417 \\ ${ }^{3}$ CEA Cadarache, Euratom Association-Cadarache, Cadarache, France \\ ${ }_{5}^{4}$ Australian National University, Canberra, Australia \\ ${ }^{5}$ FZ-Jülich, Euratom Association-FZ-Jülich, Jülich, Germany \\ ${ }^{6}$ University of California, Los Angeles, California 90095-1594 \\ ${ }^{7}$ Lawrence Livermore National Laboratory, Livermore, California 94550 \\ ${ }^{8}$ University of Wisconsin-Madison, Madison, Wisconsin 53706 \\ ${ }^{9}$ Columbia University, New York, New York 10027 \\ ${ }^{10}$ Oak Ridge National Laboratory, Oak Ridge, Tennessee 37831 \\ ${ }^{11}$ Sandia National Laboratory, Albuquerque, New Mexico 87185
}

(Received 18 November 2004; accepted 16 February 2005; published online 28 April 2005)

\begin{abstract}
A low amplitude $\left(\delta b_{r} / B_{T}=1\right.$ part in 5000) edge resonant magnetic field perturbation with toroidal mode number $n=3$ and poloidal mode numbers between 8 and 15 has been used to suppress most large type I edge localized modes (ELMs) without degrading core plasma confinement. ELMs have been suppressed for periods of up to 8.6 energy confinement times when the edge safety factor $q_{95}$ is between 3.5 and 4. The large ELMs are replaced by packets of events (possibly type II ELMs) with small amplitude, narrow radial extent, and a higher level of magnetic field and density fluctuations, creating a duty cycle with long "active" intervals of high transport and short "quiet" intervals of low transport. The increased transport associated with these events is less impulsive and slows the recovery of the pedestal profiles to the values reached just before the large ELMs without the $n=3$ perturbation. Changing the toroidal phase of the perturbation by $60^{\circ}$ with respect to the best ELM suppression case reduces the ELM amplitude and frequency by factors of 2-3 in the divertor, produces a more stochastic response in the $H$-mode pedestal profiles, and displays similar increases in small scale events, although significant numbers of large ELMs survive. In contrast to the best ELM suppression case where the type I ELMs are also suppressed on the outboard midplane, the midplane recycling increases until individual ELMs are no longer discernable. The ELM response depends on the toroidal phase of the applied perturbation because intrinsic error fields make the target plasma nonaxisymmetric, and suggests that at least some of the variation in ELM behavior in a single device or among different devices is due to differences in the intrinsic error fields in these devices. These results indicate that ELMs can be suppressed by small edge resonant magnetic field perturbations. Extrapolation to next-step burning plasma devices will require extending the regime of operation to lower collisionality and understanding the physical mechanism responsible for the ELM suppression. (C) 2005 American Institute of Physics. [DOI: 10.1063/1.1888705]
\end{abstract}

\section{INTRODUCTION}

In fusion plasmas, the fusion power production and the plasma edge conditions (the height of the electron temperature pedestal $\left.T_{e}^{\text {ped }}\right)$ are tightly coupled, requiring operation with steep edge pressure gradients that produce large, repetitive magnetohydrodynamic (MHD) instabilities known as edge localized modes (ELMs). ${ }^{1}$ These ELMs cause large, fast heat and particle impulses to the divertor target plates that can exceed the transient thermal capacity of the target plates and limit the divertor lifetime. ${ }^{2}$ In the International

\footnotetext{
a) Paper JI2 4, Bull. Am. Phys. Soc. 49, 209 (2004).

${ }^{b)}$ Invited speaker.
}

Thermonuclear Experimental Reactor (ITER), ${ }^{3}$ for example, operation with the planned fusion power gain $Q_{\text {fus }} \geqslant 10$, where $Q_{\text {fus }}$ is the ratio of fusion power production to input power, will require a high confinement mode ( $H$ mode) $T_{e}^{\text {ped }} \geqslant 4 \mathrm{keV}$. The stored energy released by an ELM, $\Delta W_{\mathrm{ELM}}$, as a fraction of the stored energy in the $H$-mode pedestal $W_{\text {ped }}$ increases with higher edge electron temperature $T_{e}^{\text {ped }}$ and lower collisionality in present devices. Consequently, an ELM in ITER is predicted to release up to $20 \%$ of the stored energy in the pedestal $W_{\text {ped }}$ to the plasma-facing components, ${ }^{4}$ where it will exceed the ablation limit for graphite by a factor of $2-4 .^{5}$ This erosion limits the divertor lifetime, contaminates the core plasma, and leads to increased tritium inventory on the plasma-facing surfaces 
when the eroded material is codeposited with hydrogenic species. $^{6}$

The ELMs, however, have beneficial effects, including the transport of fuel and impurity particles across the boundary in $H$ mode, providing a mechanism for steady-state, high performance operation with density control and reduced core impurity content. Therefore, any technique to eliminate large ELM impulses must maintain the $H$-mode edge pedestal conditions while replacing the ELM-induced transport with another, less impulsive transport process. The development of techniques and operational scenarios which significantly reduce or eliminate the impulsive loading to the divertor targets from large ELMs appears to be essential for long pulse burning plasma experiments. Consequently, the fusion community has studied several different approaches to reducing the impulsive loading to the divertor target plates caused by ELMs, including shaping the plasma to obtain smaller amplitude ELMs (e.g., type II) ${ }^{7}$ inducing ELMs before the edge pedestal stored energy $W_{\text {ped }}$ has reached the marginally stable level with external magnetic perturbations ${ }^{8}$ or pellets, ${ }^{9}$ and operation in steady-state regimes that are free of large ELM impulses, such as Quiescent $H$ mode $(\mathrm{QH} \text { mode })^{10}$ and "enhanced Dalpha mode" (EDA $H$ mode). ${ }^{11}$ These latter two regimes rely on particle transport by electromagnetic modes (edge harmonic oscillation in $\mathrm{QH}$ mode; quasicoherent mode in EDA $H$-mode) located in the pedestal to maintain density control and to avoid impurity accumulation. These regimes, however, have operational aspects which may limit extrapolation to ITER, so development of an active control scenario that is more flexible would significantly improve the viability of a burning plasma experiment.

Because ELM behavior (amplitude, frequency, and energy impulse) is sensitive to MHD stability in the boundary, which in turn depends upon the edge pressure and current profiles, a boundary stochastic layer produced by an edge resonant radial magnetic field perturbation might provide a means for active control of ELMs and the $H$-mode pedestal. The stochastic layer would enhance the cross-field transport at the foot of the $\mathrm{H}$-mode pedestal in order to hold the edge pressure gradient below the critical level for triggering the ELMs. ${ }^{12,13}$ In this paper, we report the results of using a small $(0.02 \%$ of the equilibrium field) edge resonant magnetic field perturbation from MHD control coils inside the DIII-D vacuum vessel (the "I coils"14 ) to control large ELMs in the DIII-D tokamak. We have identified two distinct operational regimes: one with an $n=3$ perturbation with a toroidal phase relative to the intrinsic error fields for which the edge stochasticity is minimized, and large ELMs are suppressed for times as long as $8.6 \tau_{E}$, where $\tau_{E}$ is the energy confinement time, without any degradation in the core plasma confinement. ${ }^{15}$ The second operational regime is obtained with a toroidal phase rotated $60^{\circ}$ from the first case which displays significant reductions in the ELM amplitude and frequency, as well as a more stochastic boundarylike plasma response. Because a $60^{\circ}$ degree phase shift of an $n$ $=3$ perturbation has no effect on an axisymmetric "target" plasma, these two regimes suggest the importance of small, intrinsic error fields on ELM dynamics in toroidal devices. In both regimes, the $H$-mode radial electric field well/transport

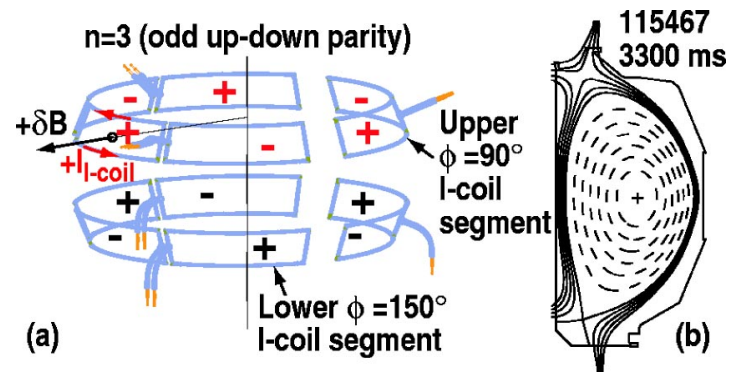

FIG. 1. (Color online). (a) The DIII-D I-coil comprises six segments above the equatorial plane (upper) and six segments below the equatorial plane (lower) centered at $60^{\circ}$ toroidal angle $\phi$ increments, starting at $30^{\circ}$. (b) The discharge shape used in these ELM suppression experiments.

barrier remains during the magnetic perturbation and the pressure at the top of the $H$-mode pedestal and the core confinement are essentially unchanged. These results demonstrate that stochastic boundary layers are compatible with $\mathrm{H}$-mode plasmas and that an edge resonant magnetic perturbation can suppress ELMs without degrading the global particle and energy confinement.

This paper is organized as follows: In Sec. II, we describe the coil system used to apply the magnetic perturbation, and the ELMing $H$-mode discharges used for these experiments. In Sec. III, we present data documenting the ELM suppression result. In Sec. IV, we present measurements showing the changes induced in the $H$-mode pedestal which might affect the stability of the MHD modes associated with the ELMs. In Sec. V we present data on the changes to edge fluctuations and cross-field transport. In Secs. VI and VII we present the dependence of the ELM suppression on the tokamak discharge safety factor at the $95 \%$ poloidal magnetic flux surface, $q_{95}$, and on the toroidal angle $\phi_{\mathrm{tor}}$, of the applied perturbation, respectively. Finally, we present our conclusions and discuss the implications of these results in Sec. VIII.

\section{EXPERIMENTAL APPROACH}

The radial magnetic field perturbations in this experiment are produced by a set of MHD mode control coils located inside the DIII-D vacuum vessel, known as the $I$ coil. ${ }^{14}$ The $I$ coil consists of 12 single-turn loops, six above and six below the midplane [Fig. 1(a)]. Each loop is constructed in a window pane geometry and mounted behind protective graphite tiles on the low field side of the vacuum vessel. These coils provide a flexible system for applying edge resonant magnetic perturbations when the coils are connected to form a toroidal mode number $n=3$ with a poloidal mode number $m$ spectrum with significant amplitudes for $8 \leqslant m$ $\leqslant 15$. This perturbation can be applied with either of two toroidal phases shifted $60^{\circ}$ with respect to each other and referred to in this paper as $\phi_{\text {tor }}$ of $0^{\circ}$ or $60^{\circ}$. Although the up/down symmetric coil pairs can be operated with the radial magnetic field in the same or opposite direction, which we refer to as "even" and "odd" parity, respectively, in this paper we consider only the odd parity case. In this configuration, the coils provide a relatively "clean" edge resonant perturbation at the $q \geqslant 3$ rational surfaces in the pedestal while hav- 
ing a relatively small impact on the plasma core. ${ }^{15}$ For most of these experiments, the DIII-D error field correction coil ( $C$ coil) which is designed to minimize the impact of known field errors in the core ${ }^{16}$ was not used because it is known to also perturb the magnetic field in the plasma boundary. ${ }^{17}$

The maximum $I$-coil current used in these experiments was $4-4.4 \mathrm{kA}, 0.25 \%$ of the plasma current. This $I$-coil current gives radial perturbation field components $\delta b_{r}^{3, m}$ of $2-3 \mathrm{G}$ on rational surfaces in the pedestal $(3 \leqslant q \leqslant 5)$, or about 1 part in 5000 of the torodial magnetic field. This $I$-coil current gives the best ELM suppression when $q_{95}$ $=3.7-3.8$, and the perturbation is applied with an $n=3$, odd parity, $\phi_{\text {tor }}=0^{\circ}$ configuration. The field line integration code TRIP3D ${ }^{17}$ predicts that the width of the edge layer where magnetic field lines are lost across the last closed flux surface by stochasticity (the "flux loss region") increases from $2 \%$ due to measured error fields to $3 \%$ with the measured error fields and $I$-coil perturbation combined, indicating that the known error fields and the $I$-coil perturbation are comparable in magnitude. This low amplitude magnetic perturbation nevertheless alters the ELM behavior dramatically.

This $n=3$ perturbation has no measurable effect on a wide range of low confinement mode ( $L$ mode) plasmas independent of parity or toroidal phase. However, the same perturbation routinely modifies ELM behavior (amplitude and frequency), although it does not always suppress large ELMs. Results with 2.2 and $4.4 \mathrm{kA}$ of $I$-coil current and slow I-coil current ramps indicate that there is no sharp threshold in applied current for ELM modification as might be expected, e.g., for triggering an instability threshold or island overlap. These results suggest that the plasma response to the edge stochastic field (which is not modeled by field line integration codes such as TRIP3D) is important. Amplification of the $I$-coil "seed" perturbation may occur for plasmas near marginal stability, such as ELMing $H$-mode plasmas, as predicted by Boozer. ${ }^{18}$

The discharges for these experiments had a weak updown asymmetric equilibrium biased downward by $2.0 \mathrm{~cm}$, creating a primary $X$ point in the lower divertor [Fig. 1(b)]. Reproducible ELM suppression has been obtained in this shape with $B_{T}=1.6 \mathrm{~T}$ and $I_{p}=1.1 \mathrm{MA}\left(q_{95}=3.8\right)$, with $B_{T}$ $=2.0 \mathrm{~T}$ and $I_{p}=1.4 \mathrm{MA}\left(q_{95}=3.8\right)$, and with $B_{T}=2.0 \mathrm{~T}$ and $I_{p}=1.1 \mathrm{MA}\left(q_{95}=4.9\right)$. ELM suppression has also been obtained in the ITER scenario 2 shape. ${ }^{15}$ Table I summarizes the discharge shaping and plasma parameters for the discharges reported on in this paper.

\section{SUPPRESSION OF THE IMPULSIVE LOADING TO THE DIVERTOR TARGET}

Application of an odd parity, $\phi_{\text {tor }}=0^{\circ} n=3 I$-coil perturbation with a current of $4.4 \mathrm{kA}$ during the flattop of a steadystate, high performance ELMing $H$-mode results in suppression of most large ELMs without any degradation in confinement, as shown in Fig. 2. The suppression of large ELM impulses on the $D_{\alpha}$ recycling light from the lower, primary divertor [Fig. 2(a)] is compared to the ELMing behavior in an identical reference discharge except for an I-coil current of 0 kA [Fig. 2(b)]. Several isolated large ELMs re-
TABLE I. Summary of shape and plasma parameters for discharges in this paper.

\begin{tabular}{lccc}
\hline \hline & 115467 & 119390 & 119854 \\
\hline$I_{p}(\mathrm{MA})$ & 1.13 & 1.13 & 1.4 \\
$B_{T}(\mathrm{~T})$ & 1.6 & 1.6 & 2.0 \\
$\kappa$ & 1.8 & 1.8 & 1.7 \\
$\delta_{\text {up }}$ & 0.35 & 0.35 & 0.39 \\
$\delta_{\text {low }}$ & 0.73 & 0.73 & 0.75 \\
$A$ & 3.1 & 3.1 & 3.1 \\
$q_{95}$ & 3.75 & 3.73 & 3.80 \\
$n_{e}\left(\mathrm{~m}^{-1}\right)$ & $6.9 \times 10^{19}$ & $7 \times 10^{19}$ & $7.7 \times 10^{19}$ \\
$P_{\text {inj }}(\mathrm{MW})$ & 5.1 & 4.9 & 4.9 \\
$W_{\mathrm{MHD}}(\mathrm{MJ})$ & 0.71 & 0.73 & 1.07 \\
$\tau_{E}(\mathrm{~ms})$ & 163 & 141 & 211 \\
$H_{\mathrm{L} 89}$ & 2.1 & 1.9 & 2.2 \\
$\beta_{N}$ & 2.3 & 2.1 & 2.18 \\
$n_{e}^{\text {ped }} / n_{\mathrm{GW}}$ & 0.57 & 0.46 & 0.42 \\
$\nu_{\text {ped }}^{*}$ & 0.56 & 0.85 & 0.38 \\
$\beta^{\text {ped }}$ & $1.1 \%$ & $0.7 \%$ & $1.4 \%$ \\
$\rho_{\text {ped }}^{*}$ & 0.49 & 0.40 & 0.42 \\
\hline \hline
\end{tabular}

main, as seen in Fig. 2(a). To date we have achieved ELM suppression for times as long as $8.6 \tau_{E}(>1 \mathrm{~s})$ in similar discharges. ${ }^{15}$ The global particle and power balance are not altered by the ELM suppression, as indicated by the similar evolution of the plasma density and gas fueling [Fig. 2(c)], and of the plasma stored energy [Fig. 2(d)] in these two discharges. These results suggest that the impulsive transport from the large type I ELMs has been replaced by another less impulsive transport process. Also shown in Fig. 2(e) is the electron pedestal pressure, which increases about $20 \%$ during the $I$-coil pulse. This is important for maintaining a similar level of core plasma confinement.

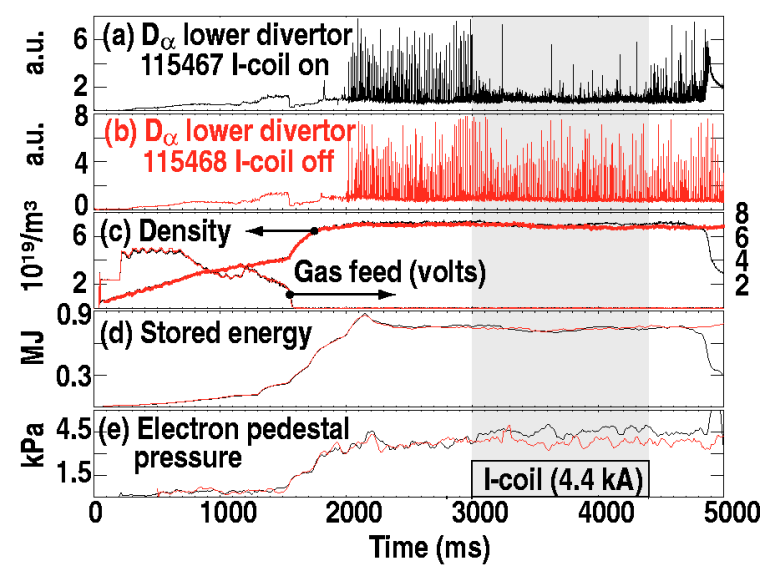

FIG. 2. (Color online). Comparison of ELM suppression and core confinement in identical discharges with (115467, black) and without (115468, gray) an $n=3$ odd parity $\phi_{\text {tor }}=0^{\circ} I$-coil pulse of $4.4 \mathrm{kA}$ : $D_{\alpha}$ recycling in the lower divertor for (a) an $I$-coil current $=4.4 \mathrm{kA}$ from 3000 to $4400 \mathrm{~ms}$ (shaded) and (b) an identical reference discharge with $I$-coil current $=0 \mathrm{kA}$. The plasma density and gas feed (c) and the plasma stored energy (d) for the ELM suppression discharge (black) and the I-coil off reference discharge (gray). (e) The electron pedestal pressure during the $I$-coil pulse (black) relative to the $I$-coil off reference (gray). 


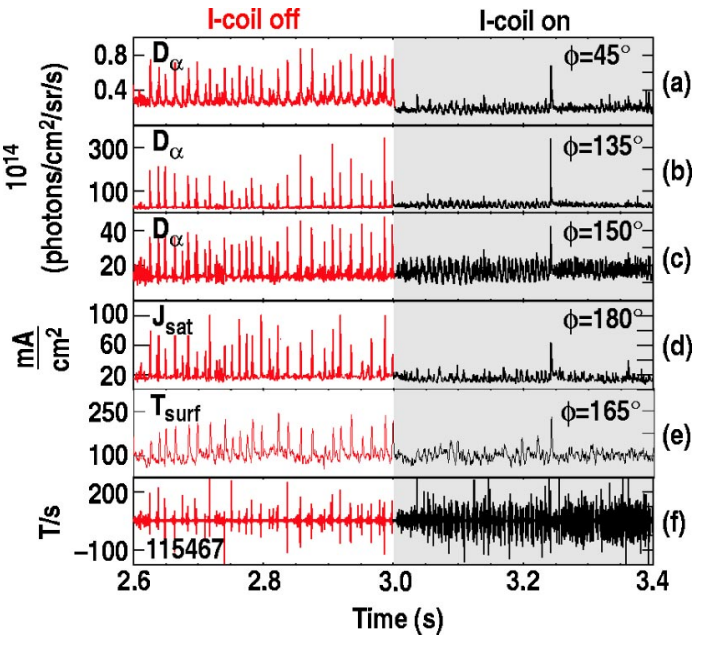

FIG. 3. (Color online). Expanded view of the onset of ELM suppression at $3000 \mathrm{~ms}$ in discharge 115467 of Fig. 2 on various poloidally and toroidally distributed boundary diagnostics, including $D_{\alpha}$ recycling from the outboard midplane (a), the lower divertor (b), and the upper divertor (c); particle flux to the lower divertor strike point (d); surface temperature variation at the lower, outer strike point (e); and magnetic fluctuations from the plasma edge (f). The toroidal angle $\phi$ of the measurement is indicated in (a-e).

An expanded view of the onset of ELM suppression in discharge 115467 at $3000 \mathrm{~ms}$ is shown in Fig. 3. The ELM suppression evident in the lower divertor recycling [Fig. 2(a) versus Fig. 2(b)] is global and readily apparent on all recycling measurements (outer midplane [Fig. 3(a)], lower [Fig. 3(b)] and upper [Fig. 3(c)] divertor, and inner wall/centerpost (not shown)). The ELM suppression is also seen in the particle flux to the primary lower divertor measured by Langmuir probes in the divertor tiles [Fig. 3(d)] and in the variation of the surface temperature $T_{\text {surf }}$ of the divertor target near the outer strike point measured by an infrared camera in fast $(100 \mu \mathrm{s})$ line scan mode [Fig. 3(e)]. These measurements are distributed both poloidally and toroidally around the machine, indicating that the suppression is indeed global. The ELMs are suppressed within one ELM cycle (about $16 \mathrm{~ms}$ ) of turning on the $I$ coil at $3000 \mathrm{~ms}$. The first isolated ELM at $3245 \mathrm{~ms}$ is seen on all these diagnostics to have comparable magnitude and duration as the ELMs before the I-coil pulse, suggesting that the discharge has not simply integrated the stored energy and particle content that would have been lost during the ELMs, but that ELM-induced transport has been replaced by another transport process which is seen in Fig. 3 as an increase in broadband magnetic field [Fig. 3(f)] and recycling fluctuations modulated by a $130 \mathrm{~Hz}$ oscillation most apparent in the upper divertor $D_{\alpha}$ trace [Fig. 3(c)].

The principal concern for next-step burning plasma devices such as ITER is the reduction of the impulsive heat flux to the divertor target plates. ${ }^{2,5}$ To quantify the change in the ELM-induced heat impulses to the target plate, radial profiles of the surface temperature were acquired by an infrared camera in fast line scan mode (1 profile/100 $\mu \mathrm{s})$. For 18 ELMs with the $I$-coil off and $18 D_{\alpha}$ oscillations with the $I$ coil on in a discharge similar to $115467, T_{\text {surf }}$ was averaged at the time

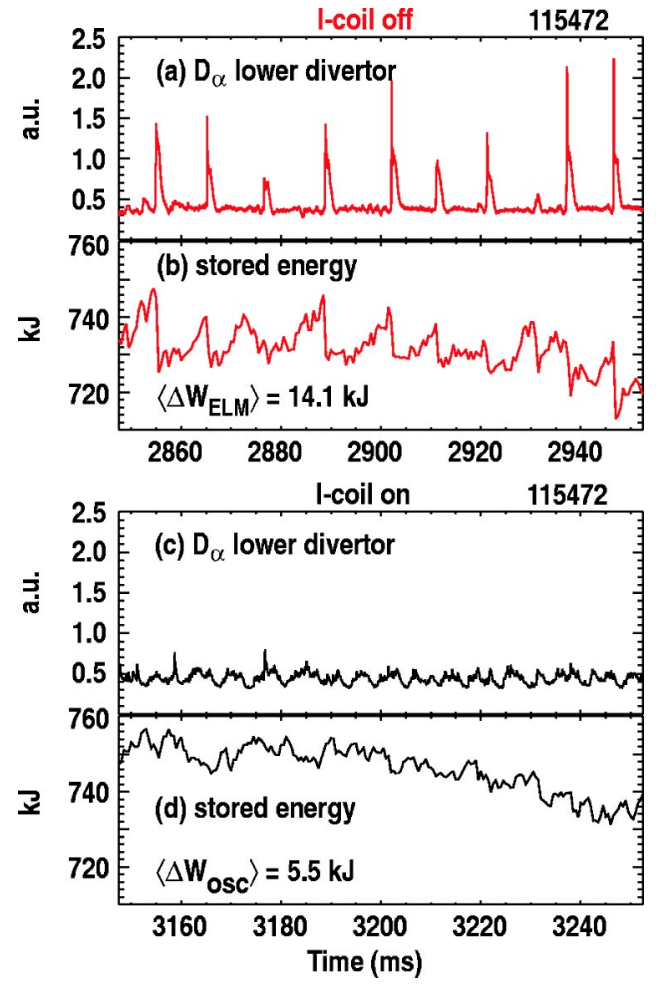

FIG. 4. (Color online). Time history of (a), (c) the lower divertor $D_{\alpha}$ and (b), (d) the plasma stored energy $W_{\mathrm{MHD}}$ in discharge 115472 with the $I$-coil off (top) and on (bottom). The average stored energy loss during type I ELMs with the $I$-coil off, $\left\langle\Delta W_{\text {ELM }}\right\rangle$, is $14.1 \mathrm{~kJ}$. The average stored energy loss during the $D_{\alpha}$ oscillations with the $I$-coil on, $\left\langle\Delta W_{\mathrm{OSC}}\right\rangle=5.5 \mathrm{~kJ}$.

of the peak $T_{\text {surf }}$ value at the divertor plate. The timeaveraged signal was then subtracted to obtain the $T_{\text {surf }}$ profile due to the impulsive power loading to the lower divertor caused by the type I ELMs (I-coil off) versus the $D_{\alpha}$ oscillations ( $I$-coil on). During the $I$-coil pulse, the $T_{\text {surf }}$ spikes are reduced below the noise level of the infrared camera, indicating that the divertor surface temperature rise due to the type I ELMs was reduced by at least a factor of 5 during the I-coil perturbation. ${ }^{12,15}$ Floor Langmuir probe measurements of the ion saturation current with a sampling rate of $40 \mu \mathrm{s}$ show a similar reduction in the impulsive particle flux (and hence convective heat flux) to the lower divertor by a factor of 8 during the $I$-coil perturbation. For both measurements, the suppression of the ELM impulses occurs across the divertor floor, indicating that the change results from a reduction in the power and particle impulses "upstream" in the pedestal, and not simply a spreading or redirection of the impulses to larger radii in the scrape-off layer (SOL). This conclusion is supported by analysis of the impulsive losses of plasma stored energy $W_{\mathrm{MHD}}$ with and without the $I$-coil perturbation. The change in $W_{\mathrm{MHD}}$ is obtained by equilibrium reconstruction with the Grad-Shafranov solver EFIT $^{19}$ in $0.5 \mathrm{~ms}$ time steps using fast magnetic probe data (Fig. 4). Typical $W_{\text {MHD }}$ drops at the ELMs $\left(\Delta W_{\text {ELM }}\right)$ are $5-23 \mathrm{~kJ}$, with an average loss $\left\langle\Delta W_{\text {ELM }}\right\rangle=14.1 \mathrm{~kJ}$ [Fig. 4(b)], compared with an average loss over a $D_{\alpha}$ oscillation $\left\langle\Delta W_{\text {OSC }}\right\rangle$ $=5.5 \mathrm{~kJ}$ during the $I$-coil pulse [Fig. 4(d)]. This factor of 2-3 drop in the stored energy loss, coupled with a four to five 


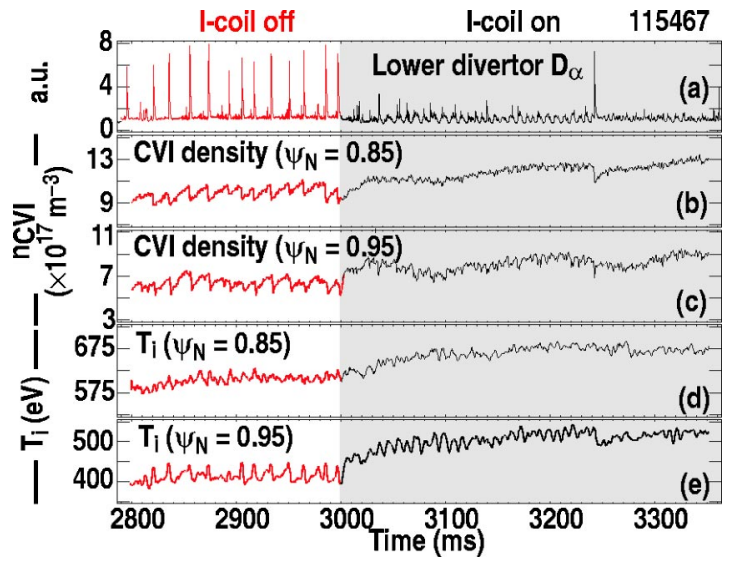

FIG. 5. (Color online). Time history of (a) lower divertor $D_{\alpha}$, carbon VI ion density at (b) $\psi_{N}=0.85$ and (c) $\psi_{N}=0.95$ in the $H$-mode pedestal, and the corresponding $T_{i}$, (d) and (e), in the $H$-mode pedestal for discharge 115467. The $I$-coil perturbation begins at $3000 \mathrm{~ms}$ (shaded region). The radial locations of the CER measurements are given in terms of the normalized poloidal magnetic flux $\psi_{N}$, where the last closed flux surface (the divertor separatrix) is $\psi_{N}=1$.

times longer time scale for the loss $(\Delta t<0.5 \mathrm{~ms}$ for the ELMs versus 2-3 ms for the $D_{\alpha}$ oscillations), gives an impulse reduction $\Delta W_{\mathrm{ELM}} / \Delta t^{1 / 2}$ by a factor of $4-7$.

\section{EFFECTS ON THE H-MODE PEDESTAL}

Electron and ion profile measurements confirm that the ELM suppression results from a change in pedestal stability and not simply a redistribution of the power flow in the scrape-off layer. In Fig. 5, we show the time evolution of the density [Fig. 5(b) and 5(c)] and temperature [Fig. 5(d) and 5(e)] of carbon VI (CVI) ions in the $H$-mode pedestal measured with charge exchange recombination spectroscopy (CER) with $0.5 \mathrm{~ms}$ integration time. For the pedestal conditions in these plasmas, the carbon VI ion temperature is well equilibrated with the deuterium working ions. During the ELMing phase, the pedestal shows primarily density drops at the ELMs, indicating that the ELM energy loss is primarily due to convection rather than conduction. The density drops associated with the ELMs are suppressed within a single ELM cycle after the turn-on of the $I$-coil perturbation at $3000 \mathrm{~ms}$. In contrast, the less regular $T_{i}$ drops are relatively unaffected by the $I$-coil pulse. Electron density $n_{e}$ and temperature $T_{e}$ profiles, obtained by conditionally averaging the Thomson scattering profiles over the ELM cycle, show similar results. Prior to the $I$-coil perturbation, the ELMs consist nearly entirely of $n_{e}$ drops, with little discernible change in the pedestal $T_{e}$ profile. After the $I$ coil is turned on, the large ELMs are suppressed, and the ELM-induced drops in the pedestal $n_{e}$ are suppressed. At lower edge densities and collisionalities where the ELMs are more regular, with large density and temperature drops, we routinely modify the ELMs (change the amplitude and frequency) with the $I$-coil perturbation, but have not yet obtained the global suppression of the ELMs seen at higher densities and collisionalities. While this result suggests that the $I$-coil perturbation is most effective at suppressing the convective losses associated with
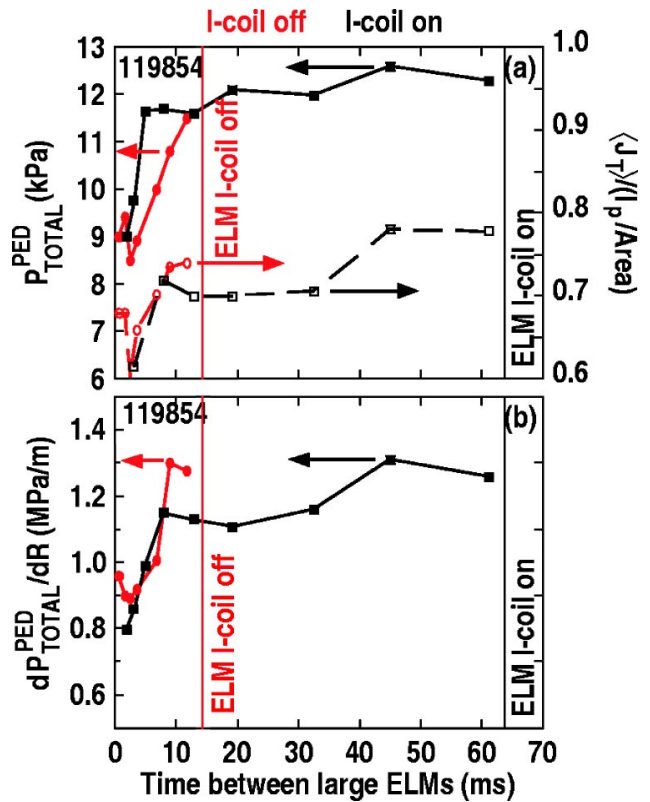

FIG. 6. (Color online). (a) Time evolution of $P_{\text {TOTAL }}^{\text {PED }}$ (filled symbols) and $\left\langle J_{T}\right\rangle / I_{p}$ /area (open symbols) over a conditionally averaged large ELM cycle with the $I$-coil off (circles) and on (squares). (b) Time evolution of $d P_{\text {TOTAL }}^{\text {PED }} / d R$ (filled symbols) with the $I$-coil off (circles) and on (squares). The data are plotted with respect to the ELM collapse time. The vertical lines at $13 \mathrm{~ms}$ and $64 \mathrm{~ms}$ correspond to the average time between successive large ELMs with the I-coil off and on, respectively.

ELMs, more experimental time is needed to optimize the ELM suppression at lower densities where the ELM loss includes a significant conduction channel.

In order to investigate how the $I$-coil perturbation alters the pedestal stability, the plasma transport code ONETwO ${ }^{20}$ was used for a discharge (119854) similar to 115467 but with more frequent isolated ELMs (every $64 \mathrm{~ms}$ on average) during the $I$-coil pulse to calculate the evolution of the total pressure profile $P_{\mathrm{TOTAL}}^{\mathrm{PED}}($ electron + ion + beam) and toroidal plasma current $\left\langle J_{T}\right\rangle$ over a representative large ELM cycle, including a correction for the measured $Z_{\text {eff. }}$. The electron and ion profiles (from Thomson scattering and CER, respectively) were conditionally sampled using the amplitude of each ELM from a midplane $D_{\alpha}$ signal to bin the profiles by phase within the ELM cycle (e.g., $D_{\alpha}$ amplitude at profile time $=(0 \%-10 \%),(10 \%-30 \%),(30 \%-60 \%)$, etc., of the peak $D_{\alpha}$ amplitude during each ELM). These individual profiles for each phase were conditionally averaged to provide the total pressure profile evolution over a representative ELM cycle without and with the $I$ coil. The average time between ELMs for this discharge was $13 \mathrm{~ms}$ with the $I$-coil off, increasing to $64 \mathrm{~ms}$ with the $I$-coil on. The resulting profiles were then parametrized using a modified hyperbolic tangent fitting routine that combines a hyperbolic tangent and a linear ramp. ${ }^{21}$ The time evolution of the total pedestal pressure $P_{\mathrm{TOTAL}}^{\mathrm{PED}}$ and the toroidal current density $\left\langle J_{T}\right\rangle$ normalized to $I_{p} /$ area, where $I_{p}$ is the measured plasma current and area is the total cross-sectional area of the discharge at the top of the $H$-mode pedestal is shown in Fig. 6(a). $\left\langle J_{T}\right\rangle$ is the peak of the total current density profile (ohmic+bootstrap+Pfirsch-Schlüter) evaluated in 


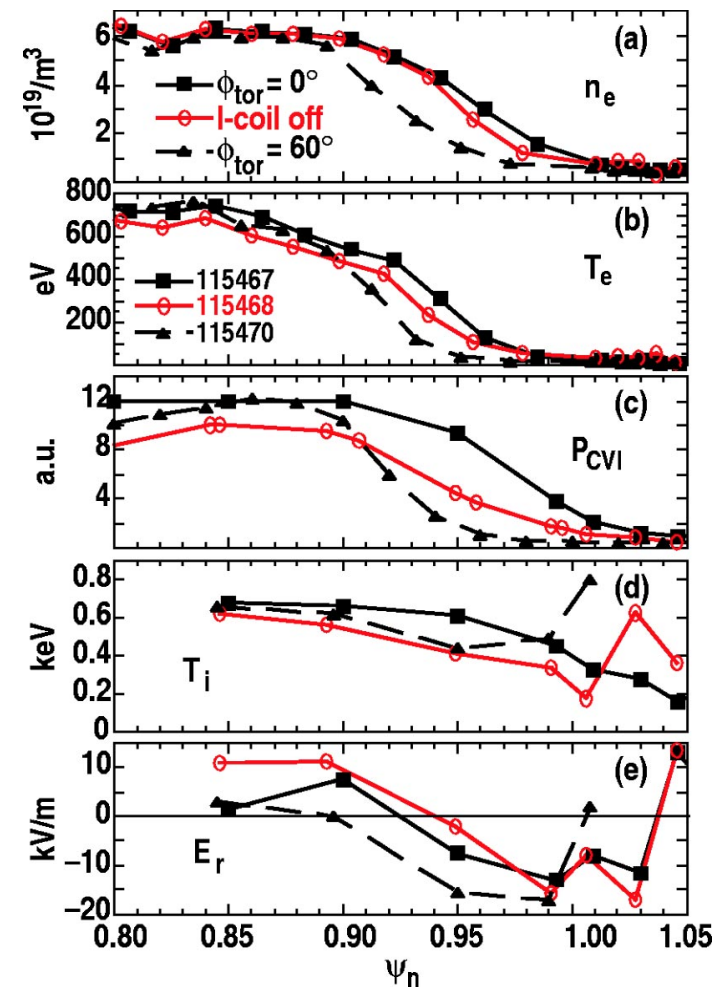

FIG. 7. (Color online). Equilibrium pedestal profiles of (a) electron density $n_{e}$, (b) electron temperature $T_{e}$, (c) carbon VI ion pressure $P_{\mathrm{CVI}}$, (d) ion temperature $T_{i}$, and (e) radial electric field $E_{r}$ at $3300 \mathrm{~ms}$ in the $I$-coil off reference discharge 115468 (open circles); in the $\phi_{\mathrm{tor}}=0^{\circ} I$-coil discharge 115467 (filled squares), and in the $\phi_{\text {tor }}=60^{\circ} I$-coil discharge (filled triangles).

ONETWO near the peak of the pressure gradient in the pedestal using the Sauter model for the bootstrap current. ${ }^{22}$ The $\left\langle J_{T}\right\rangle$ peak is generally shifted a small distance inward radially from the location of the maximum pressure gradient due to the effect of collisionality. Both $P_{\mathrm{TOTAL}}^{\mathrm{PED}}$ and $\left\langle J_{T}\right\rangle / I_{p} /$ area drop to similar levels just after a large ELM with and without the I-coil perturbation, but reach somewhat higher levels just before the ELM when the $I$ coil is on. However, the most dramatic difference in the profile evolution is the increase in the time between successive large ELMs during the I-coil pulse relative to before the $I$-coil pulse: $\tau_{\mathrm{ELM}} \approx 13 \mathrm{~ms}$ (I-coil off) and $\tau_{\mathrm{ELM}} \approx 64 \mathrm{~ms}$ (I-coil on). The gradient in the total pedestal pressure, $d P_{\text {TOTAL }}^{\text {PED }} / d R$, changes over a similar range with and without the $I$ coil, although the recovery of the gradient to the critical value is significantly slowed during the I-coil pulse by increased transport [Fig. 6(b)].

\section{ENHANCED RADIAL TRANSPORT AND FLUCTUATIONS}

An obvious choice for the origin of the increased steadystate radial transport is the effective radial transport enhancement due to the stochastic edge magnetic field. For the best ELM suppression cases to date, corresponding to an $n=3$ odd parity, $\phi_{\text {tor }}=0^{\circ}$ perturbation with $q_{95}=3.8$, however, the equilibrium pedestal profiles (Fig. 7) do not show evidence of flattening just inside the unperturbed separatrix that would be expected for strong stochasticity in this region for either

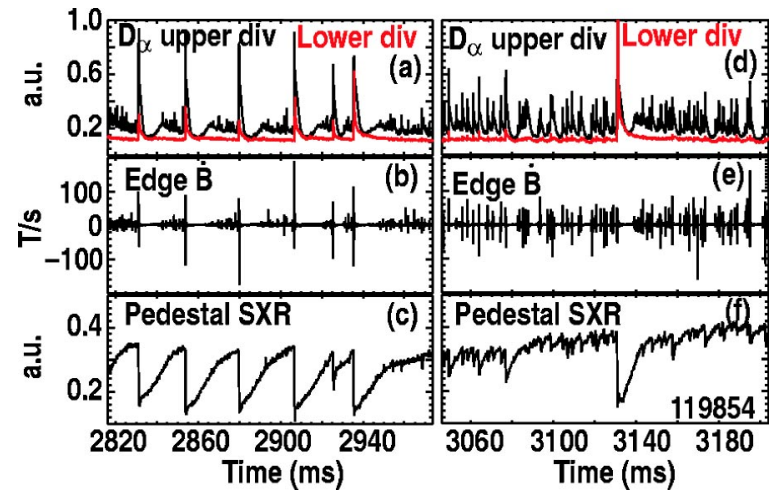

FIG. 8. (Color online). Comparison of ELM behavior with the I-coil off (left) and on (right) for discharge 119854, showing: (a) upper (black) and lower (gray) divertor $D_{\alpha}$, (b) edge magnetic fluctuations, and (c) pedestal SXR emissivity with the I-coil off and (d) upper (black) and lower (gray) divertor $D_{\alpha}$, (e) edge magnetic fluctuations, and (f) pedestal SXR emissivity with the $I$-coil on.

the electron [Figs. 7(a) and 7(b) solid lines] or ion channels [Figs. 7(c) and 7(d) solid lines]. In addition, the $H$-mode radial electric field $E_{r}$ well is maintained during the $I$-coil pulse [Fig. 7(e) solid line]. The lack of clear profile flattening is consistent with TRIP3D modeling that indicates that this perturbation at a $q_{95}=3.7-3.8$ corresponds to a minimum in the stochasticity induced in the boundary.

An alternative choice for the origin of the enhanced radial transport during the $I$-coil perturbation is an increase in the MHD activity and recycling that typically precede the large type I ELMs in these discharges without the I-coil pulse [Fig. 8(a)]. This behavior is most clearly seen in discharge 119854 which has higher $I_{p}$ and a larger number of isolated type I ELMs during the $I$-coil pulse. Both the type I ELMs and the small ELM-like events have bursts of magnetic fluctuations, but the amplitude of these fluctuations is much smaller for the small ELM-like events [Fig. 8(b)] when the $I$ coil is off. These events are often, but not always, reminiscent of type II ELMs. ${ }^{23}$ Before the $I$-coil pulse, the soft $\mathrm{x}$-ray (SXR) emissivity in the pedestal decreases about $50 \%$ at the type I ELMs, but there is little change to the SXR emissivity from the small events [Fig. 8(c)]. In contrast,

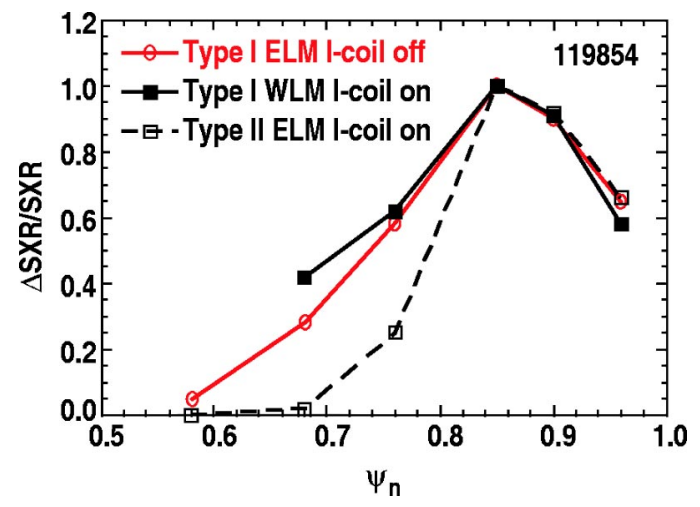

FIG. 9. (Color online). Radial profiles of the change in SXR emissivity in discharge 119854 during a type I ELM with the $I$-coil off (open circles), an isolated type I ELM with the I-coil on (filled squares), and a small (type II) ELM with the $I$-coil on (open squares). 

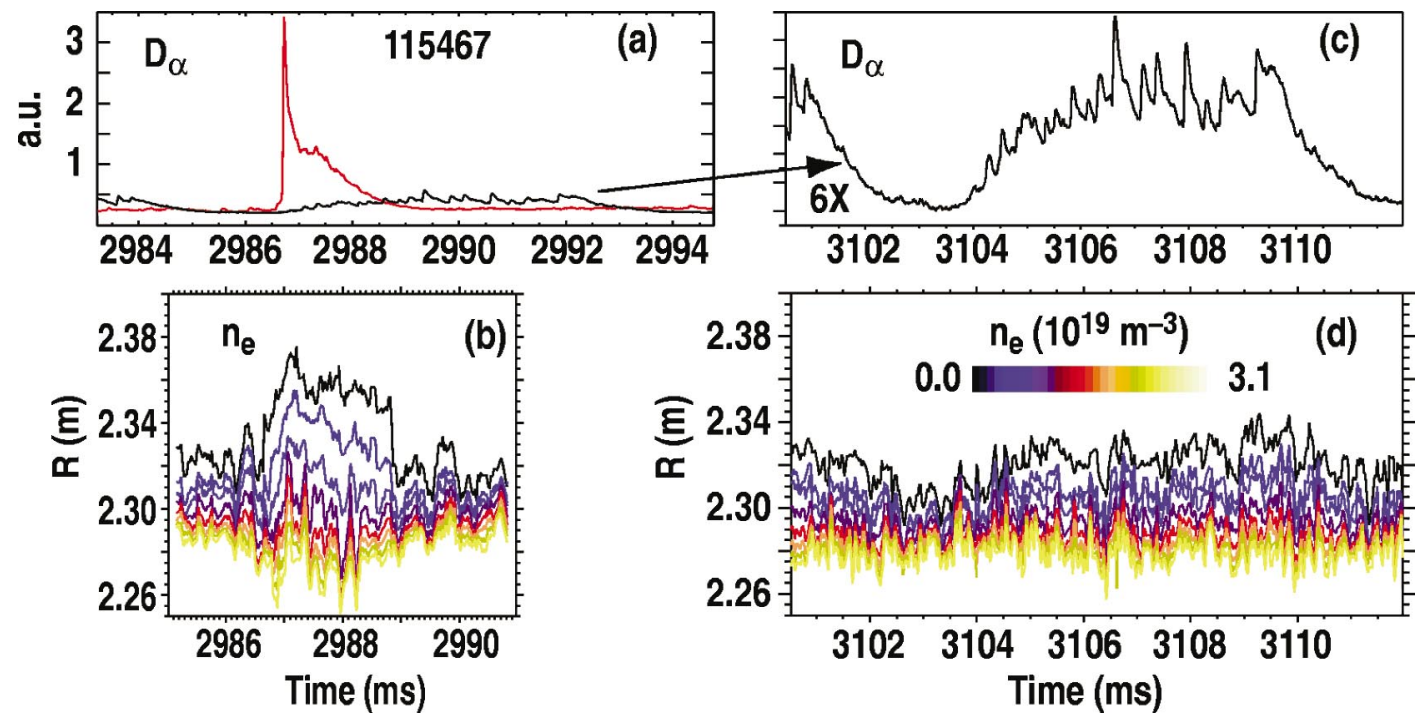

FIG. 10. (Color) Comparison of pedestal $n_{e}$ profiles measured by a swept reflectometer on the outboard midplane in discharge 115467 . (a) $D_{\alpha}$ recycling at the lower divertor strike point with the $I$-coil off (red) and on (black). (b) $D_{\alpha}$ from the lower divertor strike point during one oscillation in $D_{\alpha}$ with the $I$-coil on with the vertical scale increased $6 \times$ to show the quiet and active type II ELM intervals. Color contours of constant $n_{e}$ vs time and major radius $R$ (c) with the $I$-coil off and (d) with the $I$-coil on, showing the fluctuations in the pedestal profile during the active phase of both the ELM cycle (I-coil off) and $D_{\alpha}$ oscillation ( $I$-coil on).

when the $I$ coil is turned on, there is a large increase in the amplitude of the small ELM-like events seen in $D_{\alpha}$ [Fig. 8(d)], the magnetic fluctuations [Fig. 8(e)], and the SXR emissivity [Fig. 8(f)]. The radial extent of the SXR emissivity change $\Delta \mathrm{SXR} / \mathrm{SXR}$ due to the type I ELMs is similar
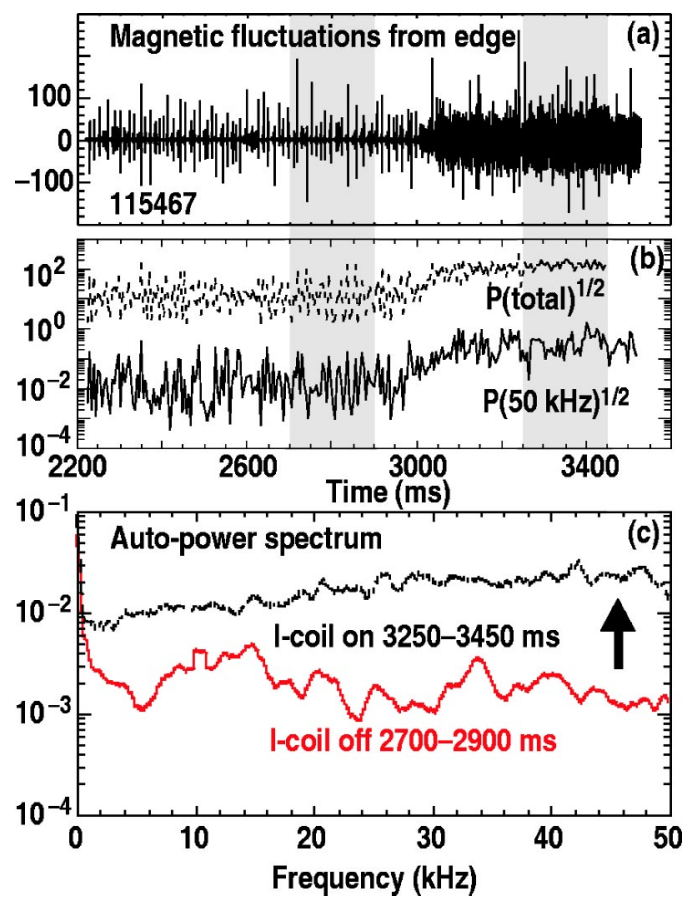

FIG. 11. (Color online). Increase in edge magnetic fluctuations as seen in time histories of (a) edge magnetic probe signal and (b) amplitude of the overall fluctuation level (dashed line) and the fluctuation level at $50 \mathrm{kHz}$ (solid line). (c) Comparison of the autopower spectra during the time intervals shaded in $(\mathrm{a}, \mathrm{b})$ showing the broadband increase in magnetic fluctuations up to the Nyquist frequency $(50 \mathrm{kHz})$ during the $I$-coil pulse (upper dashed curve) versus before the $I$-coil pulse (lower solid curve). with the $I$-coil off and on. During the $I$-coil pulse, the SXR perturbation from the small ELM-like events is more radially localized than for the type I ELMs (Fig. 9).

The change in the small ELM-like events in the primary divertor near the strike point is shown in Fig. 10(a) for a single large ELM prior to the $I$-coil pulse and for one cycle of the $D_{\alpha}$ oscillation (one group of small events) during the $I$-coil pulse, where the elapsed time $=12 \mathrm{~ms}$ and the absolute magnitude are the same for both traces. In Fig. 10(b), the $D_{\alpha}$ trace during the oscillation with the $I$-coil on is magnified $6 \times$, showing the short $(1-2 \mathrm{~ms})$ quiet interval and much longer 6-7 ms active interval consisting of a burst of small

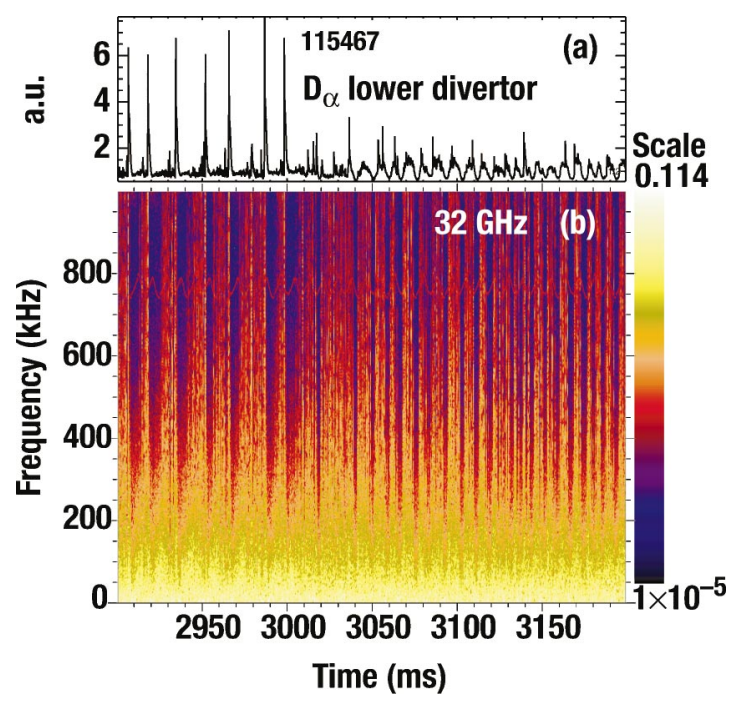

FIG. 12. (Color) (a) Lower divertor $D_{\alpha}$ and (b) color contour plot of the amplitude spectrum of density fluctuations $\tilde{n}_{e}$ measured with a $32 \mathrm{GHz}$ homodyne reflectometer, corresponding to a pedestal radius of $\psi_{N}=0.98$ and a density $n_{e}=1.3 \times 10^{19} / \mathrm{m}^{3}$. 
ELM-like activity. In contrast, the type I ELM cycle with the I-coil off [Fig. 10(a)] consists of a 1.5-2.0 ms active interval and $9 \mathrm{~ms}$ quiet interval. The fluctuations in the pedestal $n_{e}$ profile during these intervals, as measured by profile reflectometry, ${ }^{24}$ are shown in Fig. 10(c) for the I-coil off and in Fig. 10(d) for the $I$-coil on. The pedestal $n_{e}$ profile is represented by the time dependence of the major radius of contours of constant density. From Fig. 10, the pedestal profiles are similar during the quiet phase of both the large ELMs and the $D_{\alpha}$ oscillations. Type I ELMs transiently decrease the pedestal density gradient, as indicated by spreading of the density contours [Fig. 10(c)]. In contrast, during the active phase of the $D_{\alpha}$ oscillation [Fig. 10(d)], the density contours spread less, indicating that the small events decrease the pedestal gradient less than the type I ELMs without the $I$-coil pulse, but the gradient is reduced for a longer period of time than in the case of the type I ELMs. In effect, the $I$-coil perturbation has altered the pedestal dynamical state by changing the "duty cycle" of high radial transport from 2 of $11 \mathrm{~ms}$ in the case of large ELMs without the $I$ coil to 7 of $9 \mathrm{~ms}$ during the $D_{\alpha}$ oscillation with the $I$-coil on, providing a more steady-state radial transport than the type I ELMs.

This "filling in" of the quiet intervals between large ELMs is also seen in the magnetic fluctuations from the edge region (Fig. 11). In Fig. 11(a), the time history of the edge magnetic fluctuations for discharge 115467 shows this filling in after $3000 \mathrm{~ms}$ ( $I$-coil turn-on time) of the quiet intervals between the broadband bursts that occur on the leading edge of each large ELM crash before $3000 \mathrm{~ms}$. The overall increase in the magnetic fluctuation amplitude when the $I$ coil is turned on is shown in Fig. 11(b) for the total amplitude and for the amplitude of the $50 \mathrm{kHz}$ band (the Nyquist frequency $f_{N}$ ). The increase in the $50 \mathrm{kHz}$ band indicates that fluctuation amplitudes increase at frequencies beyond the bandwidth of the diagnostic. This change is evident in the autopower spectra corresponding to the shaded intervals in Figs. 11(a) and 11(b) with and without the $I$-coil pulse. During the $I$-coil pulse, the power in the edge magnetic fluctuations increases broadband up to and beyond $f_{N}=50 \mathrm{kHz}$ [Fig. 11(c)]. Fourier analysis of two high frequency magnetic coils toroidally separated on the outboard midplane indicates that this increase in magnetic fluctuations is localized to modes propagating in the direction counter to $I_{p}$ and the neutral beam momentum input. These modes can be localized to the pedestal where the toroidal rotation velocity $\nu_{\phi}$ reverses during the $I$-coil pulse. ${ }^{12}$ The higher frequency response of these coils allows us to determine that the increase in fluctuation levels extends to $75-90 \mathrm{kHz}$.

Density fluctuations in the edge without the $I$-coil pulse measured by homodyne reflectometry consist of broadband bursts (orange and yellow) during the type I ELMs alternating with quiet intervals (blue bands) correlated with the low $D_{\alpha}$ levels (Fig. 12). A striking feature of the $\widetilde{n}_{e}$ measurements is the similar qualitative features when the $I$-coil pulse is off and on, except that during the $I$-coil pulse, the discharge appears to have more rapid ELMs. These measurements are in the steep gradient region of the pedestal, away from the top of the pedestal where one would see the smaller radial

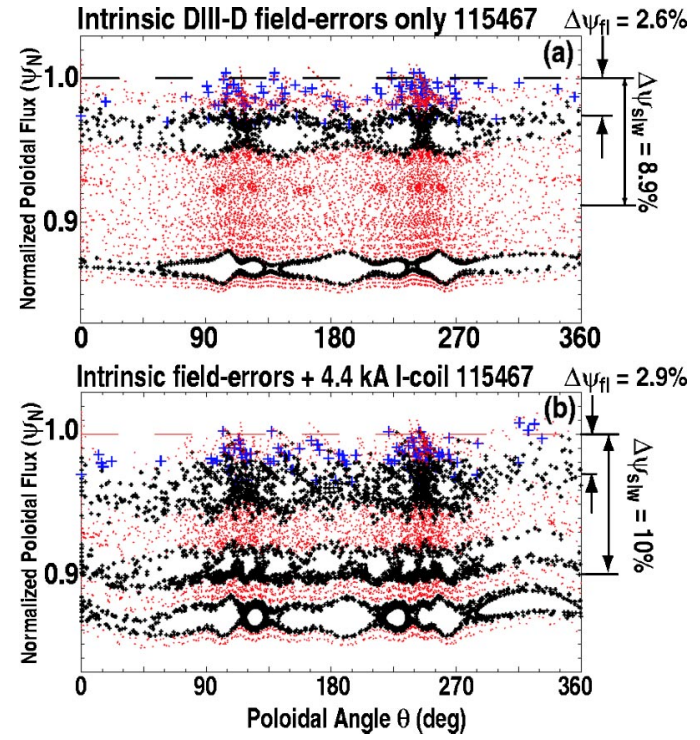

FIG. 13. (Color online). Poincare plots in the $\left(\psi_{N}, \theta\right)$ plane of the magnetic field topology in the pedestal of DIII-D discharge 115467 with (a) the measured error fields (Ref. 25) and (b) the measured error fields and the $n=3$, odd parity, $\phi_{\text {tor }}=0^{\circ} I$-coil perturbation with $4.4 \mathrm{kA}$. The width of the stochastic layer is indicated by $\Delta \psi_{s 1 w}$. In (a) the black points highlight remnant islands on the $q=3$ and $q=4$ surfaces and the large "+" indicate the innermost field line which is lost across the separatrix (dashed line), defining the width of the stochastic flux loss region, $\Delta \psi_{\mathrm{fl}}$. In (b) the black points highlight remnant islands at the $q=3, q=10 / 3$, and $q=4$ surfaces. The large + indicates the innnermost field line lost across the separatrix.

extent of the perturbations from the small events comprising the $D_{\alpha}$ oscillation [e.g., as seen in the SXR emissivity (Fig. 9)]. Measurements with beam emission spectroscopy ${ }^{25}$ confirm that the radial extent of the ELM-induced modulation of the density is more localized to the separatrix during the I-coil pulse, extending a shorter distance in toward the plasma core and a shorter distance out into the SOL.

\section{DEPENDENCE OF ELM SUPPRESSION ON $q_{95}$}

In order to demonstrate that the ELM suppression was the result of a resonant interaction with the $I$-coil perturbation, the plasma current was ramped during the $I$-coil pulse in a series of discharges to determine the optimum range of $q_{95}$ for ELM suppression. ${ }^{15}$ ELM suppression displays a resonant character as expected, with suppression limited to a narrow range in edge safety factor $3.4<q_{95}<4.0$.

TRIP3D modeling of the magnetic field topology indicates that the $n=3$ perturbation produces a stochastic layer inside the separatrix of width $\Delta \psi_{s l w}$ which is characterized by (1) stochastic magnetic field lines which wander radially across the unperturbed flux surfaces without crossing the magnetic separatrix, (2) field lines which lie in remnant islands of closed flux surfaces on the $q=(9,3),(10,3)$, and $(12,3)$ surfaces, and (3) a narrow layer of width $\Delta \psi_{\text {fl }}$ just inside the separatrix in which the stochastic field lines cross the separatrix and hit material surfaces, which we refer to as the "flux loss region" (Fig. 13).

As $q_{95}$ is changed, TRIP3D modeling indicates that the width of the flux loss region and the size and shape of remnant islands in the pedestal change. At the lower limit of the 
ELM suppression window, $q_{95} \approx 3.4$, the interaction of the $(10,3)$ and $(12,3)$ island chains created by the $I$-coil perturbation with the magnetic separatrix creates a relatively broad stochastic flux loss region of $6 \%$ in normalized poloidal flux. At the upper limit of the ELM suppression window, $q_{95}$ $\approx 4.0$, this stochastic flux loss region is $3 \%$ wide. The best ELM suppression has been obtained at $q_{95} \approx 3.7$, corresponding to the minimum width of the stochastic flux loss region of $2 \%$, and the $(9,3)$ and $(10,3)$ remnant islands located near the top of the $H$-mode pedestal. These results indicate that the best ELM suppression is obtained for the minimum stochastic field (neglecting the plasma response), and therefore may be linked to the location of the remnant islands in the pedestal. An alternative interpretation is that, in ELMing $H$ modes where the pedestal pressure and current profiles are near marginal stability for the peeling-ballooning modes associated with ELMs, significant amplification of the $I$-coil perturbation may occur. ${ }^{18}$ Present numerical models are inadequate to test such a prediction in a self-consistent calculation including the plasma response to the $I$-coil perturbation.

\section{DEPENDENCE ON TOROIDAL ANGLE OF THE APPLIED PERTURBATION}

The $q$ dependence of the ELM suppression indicates that the remnant islands formed by interaction between the error fields and the applied $I$-coil perturbation can alter the pedestal behavior. The topology of the magnetic field in the $\mathrm{H}$-mode pedestal region is compared in Fig. 13 for the measured error fields in DIII-D [Fig. 13(a)] and the combination of the measured error fields and the $n=3$, odd parity, $\phi_{\text {tor }}$ $=0^{\circ} I$-coil perturbation at $4.4 \mathrm{kA}$ [Fig. 13(b)]. The measured error fields ${ }^{26}$ produce thin, small-scale, high $k_{\theta}$ remnant island chains at the $q=3$ and $q=4$ surfaces that are distorted by higher harmonics. The addition of the $n=3 I$-coil perturbation broadens the remnant islands on the $q=3$ and $q=4$ surfaces, and drives a new island chain on the $q=10 / 3$ surface for an applied toroidal phase $\phi_{\text {tor }}=0^{\circ}$. This response depends upon the toroidal phase of the I-coil perturbation because the error fields make the "target" plasma nonaxisymmetric.

The dependence of the plasma response on $\phi_{\text {tor }}$ has been demonstrated in a series of discharges identical to 115467 except for an $I$-coil perturbation with $\phi_{\text {tor }}=60^{\circ}$. Significant ELM modification in the lower divertor [Fig. 14(a) versus the $I$-coil reference discharge in Fig. 14(b)] occurs, although more type I ELMs remain than in the $\phi_{\text {tor }}=0^{\circ}$ case [Fig. 2(a) and 2(b)]. In contrast to the $\phi_{\text {tor }}=0^{\circ}$ case where the type I ELMs are also suppressed on the outboard midplane, the midplane recycling increases until individual ELMs are no longer discernable [Fig. 14(c)]. As for the $\phi_{\text {tor }}=0^{\circ}$ case, core confinement is essentially unchanged [Fig. 14(f)].

An expanded view of the ELM modification with an $I$-coil perturbation with $\phi_{\text {tor }}=60^{\circ}$ is shown in Fig. 15. The ELM frequency and amplitude in the lower [Fig. 15(b)] and upper [Fig. 15(c)] divertors are each reduced by a factor 2-3. Similar changes to the frequency and amplitude of particle flux [Fig. 15(d)] and surface temperature [Fig. 15(e)] spikes are seen in the lower divertor. On the outer midplane, the

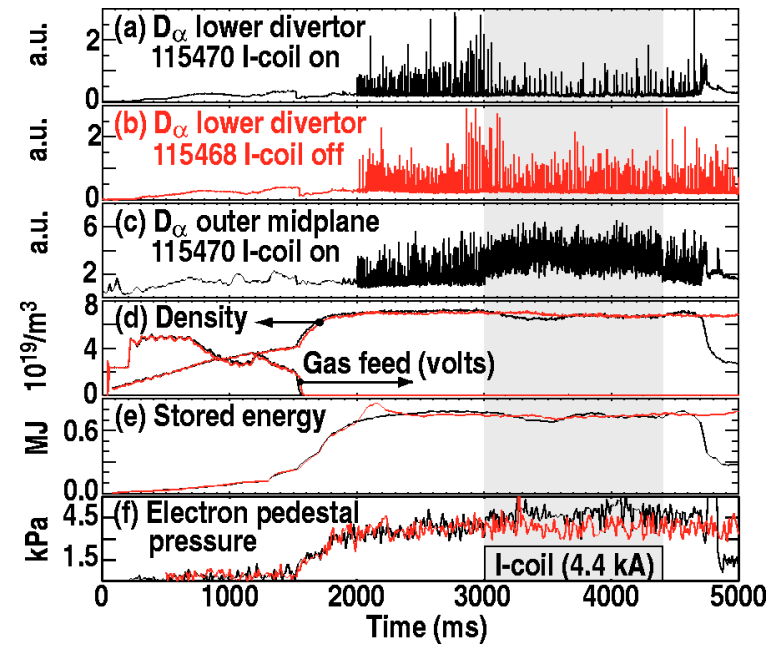

FIG. 14. (Color online). Comparison of ELM modification and core confinement in identical discharges with (115470, black) and without (115468, gray) an $n=3$ odd parity $\phi_{\text {tor }}=60^{\circ} I$-coil pulse of $4.4 \mathrm{kA}$ : $D_{\alpha}$ recycling in the lower divertor for (a) an $I$-coil current $=4.4 \mathrm{kA}$ from 3000 to $4400 \mathrm{~ms}$ (shaded) and (b) an identical reference discharge (115468) with I-coil current $=0 \mathrm{kA}$. (c) $D_{\alpha}$ recycling on the outer midplane for an $I$-coil current $=4.4 \mathrm{kA}$. The plasma density and gas feed (d) and the plasma stored energy (e) with (black) and without the I-coil perturbation (gray). (e) The electron pedestal pressure with (black) and without (gray) the I-coil pulse.

overall $D_{\alpha}$ recycling level is strongly enhanced, with the base line level between ELMs before the $I$-coil pulse rising when the I-coil is turned on to "bury" the ELMs [Fig. 15(a)], a behavior that is reminiscent of the $D_{\alpha}$ behavior in EDA $H$ mode seen in Alcator C-Mod. ${ }^{11}$ The change in $D_{\alpha}$ response when the toroidal phase is changed suggests that at least some of the variation in ELM behavior in a given device or between devices may be related to intrinsic error fields.

The response of the edge plasma profiles also differs from the $\phi_{\text {tor }}=0^{\circ}$ case, as shown by the filled triangles and

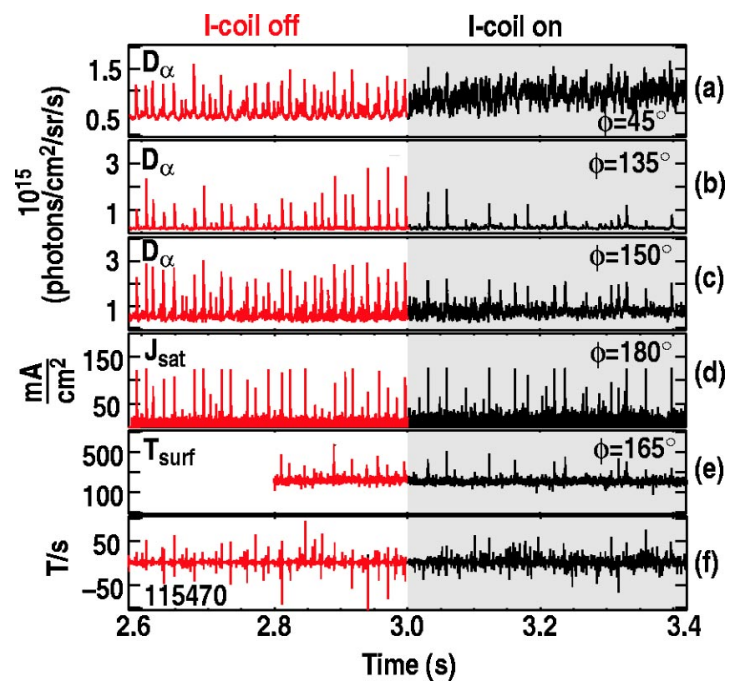

FIG. 15. (Color online). Expanded view of the onset of ELM modification at $3000 \mathrm{~ms}$ in discharge 115470 of Fig. 14, on poloidally and toroidally distributed boundary diagnostics, including $D_{\alpha}$ recycling from (a) the outboard midplane, (b) the lower divertor, and (c) the upper divertor; (d) particle flux to the lower divertor strike point; (e) surface temperature variation at the lower, outer strike point, and (f) magnetic fluctuations from the plasma edge. 
dashed lines in Fig. 7. The $n_{e}$ [Fig. 7(a)], $T_{e}$ [Fig. 7(b)], and $P_{\mathrm{CVI}}[$ Fig. 7(c)] profiles all drop in the outer pedestal for radii $\psi_{N}>0.9$. In addition, the $H$-mode $E_{r}$ well [Fig. 7(e)] shifts in by $5 \%$ in $\psi_{N}$ (filled triangles and dashed line) relative to the $\phi_{\text {tor }}=0^{\circ}$ case (filled squares and solid line). These profile changes, and the enhanced recycling at the outboard midplane are more consistent with formation of a significant stochastic layer when the $n=3 I$-coil perturbation is applied with a $\phi_{\text {tor }}=60^{\circ}$.

\section{DISCUSSION AND CONCLUSIONS}

The requirement to mitigate the heat flux impulses from large ELMs in ITER has motivated a number of different experiments aimed at achieving ELM-free $H$-mode operation (such as EDA $H$ mode and QH mode), or at controlling the impulse loading due to the ELMs with either pellets or external magnetic perturbations. Because the MHD stability of the modes associated with ELMs is determined by the pressure and current profiles in the outer portion of the $H$-mode pedestal, the use of external magnetic perturbations to enhance radial transport by forming a stochastic layer just inside the unperturbed separatrix is a logical choice. ${ }^{17}$ The enhancement of radial transport by the stochastic layer can be used to actively control the edge MHD stability by altering the pedestal pressure and current profiles, provided the coils have a mode spectrum which resonates in the edge without significantly perturbing the plasma core. Since the region of interest where the pedestal pressure and current gradients determine the ELM stability is only the last few percent in normalized poloidal flux inside the separatrix, even the relatively weak stochastic flux loss layers generated in these experiments could significantly alter ELM stability. The DIII-D I coil, although not designed for pedestal and ELM control, can provide an edge resonant perturbation with a toroidal mode number $n=3$ that only weakly perturbs core resonances.

Using the $I$-coil with an $n=3$ perturbation of $0.02 \%$ of the equilibrium magnetic field, we have examined two distinct ELM suppression regimes using $0^{\circ}$ and $60^{\circ}$ toroidal phases. For $\phi_{\text {tor }}=0^{\circ}$, large type I ELMs are suppressed for up to $8.6 \tau_{E}$ without degrading core plasma confinement. The remaining isolated ELMs have similar amplitude and time scales to the ELMs prior to the I-coil pulse. Suppression of the ELM impulses is due to a change in the pedestal stability, not just a redistribution of the ELM power and particle impulses in the SOL, as indicated by suppression of the pedestal density drops associated with the ELMs. The impulsive transport by the ELMs is replaced by an increase in small amplitude, radially narrow events with increased magnetic and density fluctuations which appear on some boundary diagnostics as rapid, small ELMs, perhaps type II ELMs. These events have a duty cycle of short "quiet" intervals of low fluctuations and transport and long "active" intervals of high fluctuations and transport, compared to the pre- $I$-coil ELM duty cycle which has short active intervals of high transport and long quiet intervals of low transport. This increased instability of small events fills in the duty cycle of transport, providing a less impulsive heat transport to the divertor target and slowing the recovery of the edge pedestal profiles to the marginally stable values for type I ELMs. Limited peeling-ballooning mode linear stability analysis with the ELITE code ${ }^{27}$ to date suggests that this delay in the recovery of the pedestal profiles to the marginally stable state is the principal effect of the $I$-coil perturbation. The growth rates and the primary toroidal mode numbers that become unstable just before the large ELMs are similar for ELMs without the $I$ coil and for the isolated ELMs that survive during the $I$-coil pulse.

Prior to the I-coil perturbation, the ELMs in these discharges consist nearly entirely of density drops, with little discernible change in the pedestal $T_{i}$ and $T_{e}$ profiles. After the $I$ coil is turned on, the large ELMs are suppressed, and the ELM-induced drops in the pedestal density are suppressed. At lower edge densities and collisionalities where the ELMs have large density and temperature drops, we routinely modify the ELMs (change the amplitude and frequency) with the $I$-coil perturbation, but have not yet obtained the global suppression of the ELMs seen at higher densities and collisionalities. While this result suggests that the $I$-coil perturbation is most effective at suppressing the convective losses associated with ELMs, more experimental time is needed to optimize the ELM suppression at lower densities where the ELM loss includes a significant conduction component.

The ELM suppression is resonant, occurring for 3.4 $<q_{95}<4$.0. TRIP3D modeling using the measured error fields indicates that this $q_{95}$ range corresponds to a minimum stochasticity for the $\phi_{\mathrm{tor}}=0^{\circ}$ phase, and to the $(9,3)$ and $(10,3)$ remnant islands lying near the top of the pedestal, subject to neglecting the plasma response. In this regime, the pedestal profiles show little evidence of a stochastic layer, consistent with the TRIP3D modeling. For $\phi_{\text {tor }}=60^{\circ}$, the ELMs are still significantly reduced, and the plasma response is more consistent with a stochastic layer, even though the TRIP3D model predicts that this perturbation should induce less stochasticity than the $\phi_{\text {tor }}=0^{\circ}$ case. This discrepancy between experimental measurements and modeling indicates that either there are sources of error fields that are not accounted for in the TRIP3D model, ${ }^{28}$ and/or that the plasma response, which cannot be modeled by a field line integration code like TRIP3D alone, significantly alters the response. The variation of both the plasma pedestal profiles and the ELM behavior when $\phi_{\text {tor }}$ is shifted $60^{\circ}$ for an $n=3$ perturbation of similar magnitude to the measured error fields further indicates that at least some of the variation in ELM behavior in a given device or between different devices may be due to the error fields in each device.

It is important to note that even for the $\phi_{\text {tor }}=60^{\circ}$ case, the large type I ELMs were still significantly modified by the $I$-coil perturbation. In addition, the same increase in small scale, type II ELM-like activity is seen in these discharges. The ELM suppression can therefore most likely be further optimized at this toroidal phasing. The $I$ coils were not designed for ELM control, and provide a relatively weak perturbing field in the boundary for the maximum I-coil current available to date $(4.4 \mathrm{kA})$. Future experiments will explore the ELM modification/suppression at increased perturbation 
field amplitude. This current should allow a strong stochastic layer to be formed for either $\phi_{\text {tor }}$, and will allow us to explore the relative importance of the remnant islands at the top of the $H$-mode pedestal and the stochastic flux loss layer at the foot of the pedestal on the effectiveness of the ELM suppression.

These results suggest an exciting possibility for controlling ELMs in next-step burning plasma devices such as ITER. The suppression is reproducible over a range of plasma shapes provided they have the appropriate resonant $q\left(3.4 \leqslant q_{95} \leqslant 4.0\right)$. The level of perturbing field required is small, only 1 part in 5000 on the resonant surfaces in the pedestal. This field produces a substantial reduction to the impulsive load to the divertor while maintaining the core confinement and pedestal pressure. However, to date ELM suppression has only been obtained for collisionalities 0.38 $<\nu^{*}<0.8$ which are relatively high compared to ITER $\left(\nu_{\mathrm{ITER}}^{*} \approx 0.06\right)$. As collisionality is lowered (e.g., by pumping), the size and radial extent of the type I ELM, $\Delta W_{\mathrm{ELM}} / W_{\mathrm{PED}}$ increase. The broader radial extent of the ELM will require a larger $I$-coil perturbation $\delta b_{r} / B_{T}$. A larger perturbing field will also make separating the effects of the intrinsic error fields and the applied perturbation easier. Extrapolating ELM suppression to ITER conditions requires additional experimental time to optimize the $I$-coil current and configuration (e.g., $\phi_{\text {tor }}$ and parity), and to determine the underlying physics. It is likely, however, that a model of the ELM suppression that can be extrapolated with confidence to ITER $H$-mode pedestal conditions will require not only additional experimental results, but improved models for calculating the self-consistent plasma response to edge resonant magnetic perturbations in poloidally diverted tokamaks. These computational models will need to include both the relevant MHD stability and the stochasticity-enhanced radial transport. While development of a fully self-consistent model of the plasma equilibrium, stability and transport in the 3D boundary created by the edge resonant magnetic perturbation is a daunting task, the payoff lies in an increased understanding of the interplay among the plasma physics elements governing the $H$-mode pedestal. This understanding will aid future devices, whether or not the use of edge resonant perturbations to control ELMs scales successfully to ITER.

\section{ACKNOWLEDGMENTS}

This work was funded by the U.S. Department of Energy under Grant Nos. DE-FC02-04ER54698, DE-FG0204ER54758, DE-FG03-01ER54615, W-7405-ENG-48, DEFG03-96ER54373, DE-FG02-89ER53297, DE-AC0500OR22725, and DE-AC04-94AL85000. The authors wish to thank the DIII-D Tokamak Operations Group for maintaining and operating the device for these experiments. In addition, they thank the respective foreign funding agencies for supporting participation by their international colleagues in these experiments.

${ }^{1}$ A. Loarte, M. Becoulet, G. Saibene et al., Plasma Phys. Controlled Fusion 44, 1815 (2002).

${ }^{2}$ G. Federici, P. Andrew, P. Barabaschi et al., J. Nucl. Mater. 313-316, 11 (2003).

${ }^{3}$ ITER Physics Basis Editors, ITER Physics Expert Group Chairs and CoChairs, and ITER Joint Central Team and Physics Integration Unit, Nucl. Fusion39, 2137 (1999).

${ }^{4}$ A. Loarte, G. Saibene, R. Sartori et al., Plasma Phys. Controlled Fusion 45, 1549 (2003).

${ }^{5}$ G. Federici, A. Loarte, and G. Strohmayer, Plasma Phys. Controlled Fusion 45, 1523 (2003).

${ }^{6}$ G. Federici, C. H. Skinner, J. N. Brooks et al., Nucl. Fusion 41, 1967 (2001).

${ }^{7}$ S. Saarelma, G. Gunter, and L. D. Horton, Nucl. Fusion 43, 262 (2003).

${ }^{8}$ M. Mori, H. Aikawa, Y. Asahi et al., Proceedings of the 14th IAEA Conference on Plasma Physics and Controlled Fusion, Würzburg, edited by IAEA Editorial Staff (IAEA, Vienna, 1993), Vol. 2, p. 567.

${ }^{9}$ P. T. Lang, J. Neuhauser, L. D. Horton et al., Nucl. Fusion 43, 1110 (2003); P. T. Lang, G. D. Conway, T. Eich et al., ibid. 44, 665 (2004).

${ }^{10}$ K. H. Burrell, M. E. Austin, D. P. Brennan et al., Phys. Plasmas 8, 2153 (2001).

${ }^{11}$ M. Greenwald, R. Boivin, P. Bonoli et al., Plasma Phys. Controlled Fusion 42, A263 (2000); A. Mazurenko, M. Porkolab, D. Mossessian, J. A. Snipes, X. Q. Xu, and W. M. Nevins, Phys. Rev. Lett. 89, 225004 (2002).

${ }^{12}$ T. E. Evans, R. A. Moyer, P. R. Thomas et al., Phys. Rev. Lett. 92, 235003-1 (2004).

${ }^{13}$ M. Bécoulet, G. Huysmans, P. Thomas et al., "Nonlinear heat transport modeling with edge localized modes and plasma edge control in tokamaks," presented at the 20th IAEA Fusion Energy Conference, Vilamoura, Portugal [Nucl. Fusion (submitted)].

${ }^{14}$ G. L. Jackson, P. M. Anderson, J. Bialek et al., Proceedings of the 30th European Physical Society Conference on Controlled Fusion and Plasma Physics, St. Petersburg, Russia, 2003, edited by R. Koch and S. Lebedev (European Physical Society, Geneva, 2003), CD-ROM, Paper P-4.47.

${ }^{15}$ T. E. Evans, R. A. Moyer, J. G. Watkins et al., "Suppression of large edge localized modes with a stochastic magnetic boundary in high confinement DIII-D Plasmas," presented at the 20th IAEA Fusion Energy Conference, Vilamoura, Portugal [Nucl. Fusion (submitted)].

${ }^{16}$ M. Okabayashi, J. Bialek, M. S. Chance et al., Phys. Plasmas 8, 2071 (2001).

${ }^{17}$ T. E. Evans, R. A. Moyer, and P. Monat, Phys. Plasmas 9, 4957 (2002).

${ }^{18}$ A. H. Boozer, Phys. Plasmas 10, 1458 (2003).

${ }^{19}$ L. L. Lao, H. E. St. John, R. D. Stambaugh, A. G. Kellman, and W. Pfeiffer, Nucl. Fusion 25, 1611 (1985).

${ }^{20}$ H. E. St. John, Plasma Phys. Control. Nucl. Fusion Research 1994, Proceedings of the 15th International Conference, Seville, 1994, edited by IAEA Editorial Staff (IAEA, Vienna, 1995), Vol. 3, p. 603.

${ }^{21}$ R. J. Groebner, D. R. Baker, K. H. Burrell et al., Nucl. Fusion 41, 1789 (2001).

${ }^{22}$ O. Sauter, C. Angioni, and Y. R. Lin-Liu, Phys. Plasmas 6, 2834 (1999).

${ }^{23} \mathrm{~T}$. H. Osborne (private communication).

${ }^{24}$ L. Zeng, E. J. Doyle, T. L. Rhodes, G. Wang, W. A. Peebles, and K. H. Burrell, Rev. Sci. Instrum. 74, 1530 (2003); G. Wang, L. Zeng, E. J. Doyle, T. L. Rhodes, and W. A. Peebles, ibid. 74, 1525 (2003).

${ }^{25}$ G. R. McKee, R. Ashley, R. Durst, R. Fonck, M. Jakubowski, K. Tritz, K. H. Burrell, C. M. Greenfield, and J. Robinson, Rev. Sci. Instrum. 70, 913 (1999).

${ }^{26}$ J. L. Luxon, M. J. Schaffer, G. L. Jackson, J. A. Leuer, A. Nagy, J. T. Scoville, and E. J. Strait, Nucl. Fusion 43, 1813 (2003).

${ }^{27}$ P. B. Snyder, H. R. Wilson, J. R. Ferron, L. L. Lao, A. W. Leonard, D. Mossessian, M. Murakami, T. H. Osborne, A. D. Turnbull, and X. Q. Xu, Nucl. Fusion 44, 320 (2004).

${ }^{28}$ T. E. Evans and R. A. Moyer, J. Nucl. Mater. 313-316, 1282 (2003). 